\title{
Highlights in Multiple Myeloma Treatment from EHA2021
}

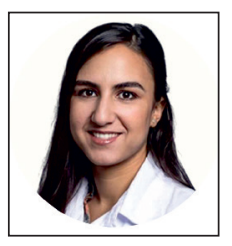

Dr Carmen de Ramon Ortiz

Division of Hematology

University Hospital of Geneva (HUG)

Geneva, Switzerland
DOI: 10.36000/HBT.OH.2021.09.050

De Ramon Ortiz C. Highlights in Multiple Myeloma Treatment from EHA2021. healthbook TIMES Onco Hema. 2021;(9):56-58.

\section{A new quadruplet regimen for the treatment of NDMM}

At EHA2021, Prof. Katja Weisel presented the updated interim analysis of the phase II GMMG-CONCEPT trial that aimed to investigate isatuximab plus carfilzomib, lenalidomide and dexamethasone (Isa-KRd) in patients with high-risk newly diagnosed multiple myeloma (NDMM). ${ }^{1}$ The trial was conducted in 20 German centers and included 153 patients with highrisk newly diagnosed multiple myeloma (NDMM). Eligible were patients with high-risk disease, defined by either del(17p), t(4;14), $\mathrm{t}(14 ; 16)$, or $>3$ copies of $1 \mathrm{q} 21$, together with an International Staging System (ISS) stage II or III. In addition, patients were required to have adequate organ function and no more than one prior anti-myeloma treatment. Transplant-eligible patients $(n=117)(\operatorname{arm} A)$ received 6 cycles of Isa-KRd induction, 4 cycles of Isa-KRd consolidation and Isa-KR maintenance, while patients who were transplant-ineligible or $\geq 70$ years of age $(n=36)(a r m B)$ received 2 additional cycles of Isa-KRd. The primary endpoint was minimal residual disease (MRD: threshold: $10^{-5}$ ) negativity after consolidation and the secondary endpoint was progression-free survival (PFS). In the present report, data of the first 50 patients are presented (arm $\mathrm{A}: \mathrm{n}=46$; arm $\mathrm{B}: \mathrm{n}=4$ ). At baseline, high-risk cytogenetics including del(17p), t(14;4), t(14;16) and $>3$ copies of 1 q21 were present in 52\%,38\%,12\% and $42 \%$ of patients, respectively. After 6 induction cycles, the overall response rate (ORR) was $100 \%$, with $90 \%$ of patients in arm A achieving very good partial response (VGPR) or better, and 46\% achieving a complete response (CR)/stringent CR. After a median follow-up of 24.9 months, the median PFS of the total population was not reached, with PFS rates of $79.6 \%$ after 12 months and $75.5 \%$ after 24 months. Regarding safety, the most common treatmentemergent adverse events (TEAEs) of grades 3 and 4 were mostly hematological and included neutropenia (34\%), Iymphopenia (28\%) and leukopenia (26\%). Conclusively, the quadruplet treatment regimen of Isa-KRd is an attractive potential treatment option as a new standard of care (SOC) for patients with NDMM. However, a longer follow-up of these patients is required to confirm the results.

\section{Diagnostic Biomarker in Transplant-eligible Multiple Myeloma} Achieving better outcomes for multiple myeloma (MM) patients is not solely dependent on improving treatment options but also on developing diagnostic tools. An interesting study on circulating tumor cells (CTCS) as a diagnostic biomarker in transplant-eligible MM patients was presented by Dr Juan José Garcés. ${ }^{2}$ The Spanish GEM2012 trial included transplanteligible MM patients with available CTCs assessment $(n=374)$. CTCS were determined in peripheral blood by next-generation flow cytometry (sensitivity: $2 \times 10^{-6}$ ) and displayed as \% CTC out of total peripheral blood (PB) leukocytes. Transplant eligible patients were given bortezomib plus lenalidomide plus dexamethasone (VRd) for 6 cycles followed by autologous stem cell transplant (ASCT) conditioned with busulfan plus melphalan versus melphalan and posttransplant consolidation with 2 cycles of VRd. Patients were stratified into three risk groups according to their CTC percentage: 0\%, $<0.24 \%, \geq 0.24 \%$ CTCs. Both PFS and overall survival (OS) outcomes are significantly better in the patient group with $0 \% \mathrm{CTCS}$, resembling the great prognostic potential of CTCS as a biomarker. The PFS was not reached in the 0\% CTCs patient group while it was 78 months and 44 months in the patient groups with $<0.24 \%$ and $\geq 0.24 \%$ CTCs. Accordingly, OS rate at 5 -years was 100\%, 81\%, and $67 \%$ for patients with $0 \%,<0.24 \%$ and $\geq 0.24 \%$ CTCs. Interestingly, patients who do not achieve complete response or better ( $\geq C R)$ or MRD-negativity but do have undetectable (O\%) CTCs, still show high PFS rates. Additionally, among patients showing $\geq C R$ and MRD-negativity, patients with 0\% CTCs achieve superior outcomes. Similarly, patients with 0\% CTCs at the beginning of treatment achieved better outcomes. Therefore, elevated CTC levels predict poor survival regardless of other baseline prognostic factors.

In the same Spanish GEM2012 study, the sensitivity of mass spectrometry (MS) versus serum and protein electrophoresis (SPEP) after immunofixation (IFE) for detection of the monoclonal component $(\mathrm{MC})$ as a biomarker for disease progression in $\mathrm{MM}$ 


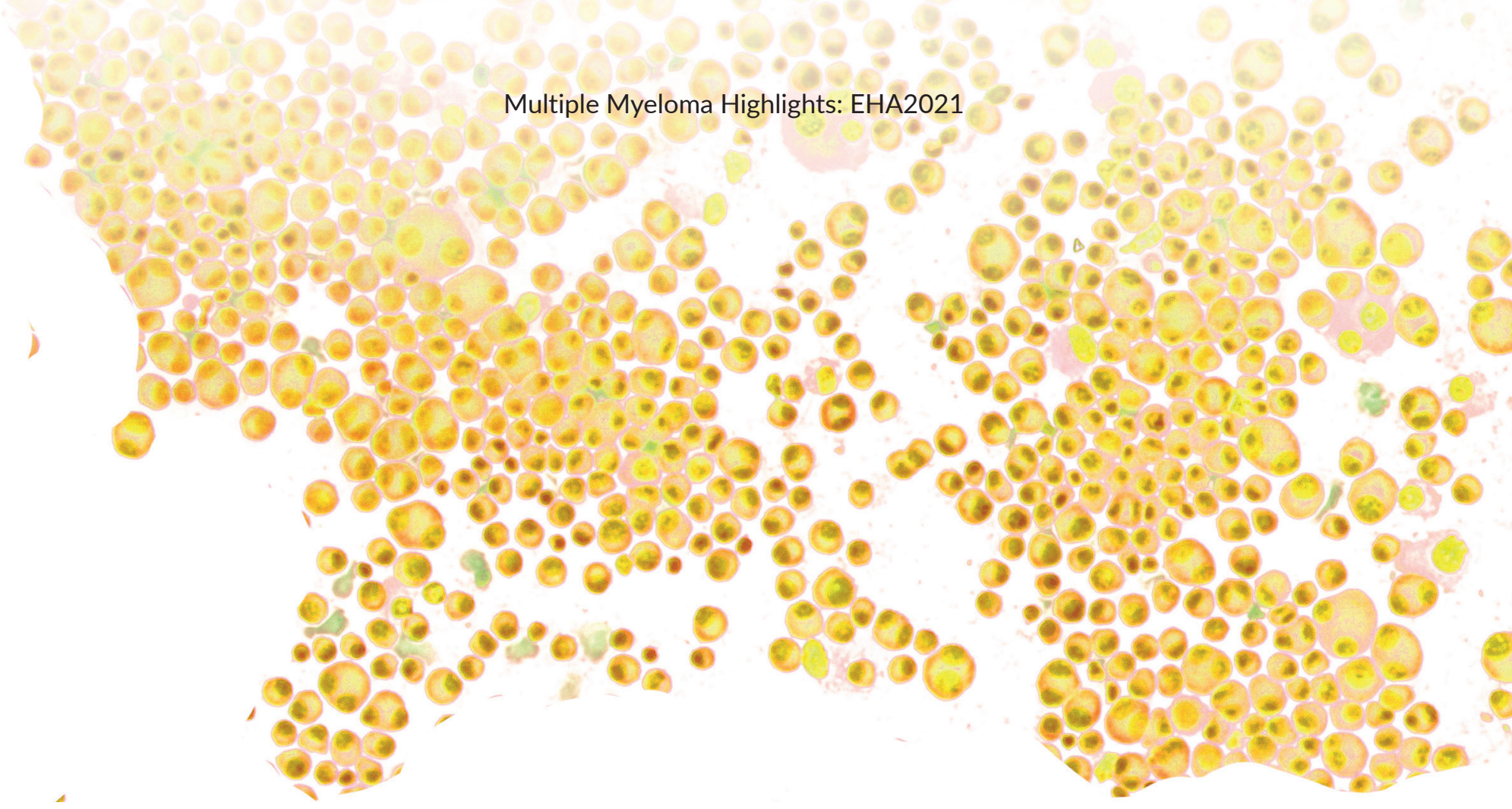

was assessed. ${ }^{3}$ MS and SPEP/IFE were performed at three different time points: after induction therapy with 6 cycles of VRd after ASCT and after 2 cycles of VRd-consolidation. At all three time points, MS yielded a higher percentage of patients with detectable MC than SPEP/IFE (63\% vs 52\% post-induction, $46 \%$ vs $36 \%$ post-ASCT, and $35 \%$ vs $27 \%$ post-consolidation). Complete response (CR) was defined as undetectable MC. Patients who achieved CR after consolidation by both SPEP/ IFE and MS assessment showed a significantly higher PFS than those who showed undetectable MC by SPEP/IFE but not by MS analysis. Conclusively, MS identified the presence of paraprotein in a higher proportion of patients throughout the screening and identified the residual disease in a cohort of patients in standard CR but at increased risk of progression.

\section{New triplet regimen for transplant-ineligible patients with NDMM}

At EHA2021, Prof. Thierry Facon presented updated efficacy and safety results from a prespecified interim overall survival (OS) analysis of the multicenter, randomized, MAIA trial assessing daratumumab plus lenalidomide and dexamethasone (Rd) versus $\mathrm{Rd}$ alone in transplant-ineligible patients with NDMM. Overall, 737 patients were randomized 1:1 to receive either daratumumab $(16 \mathrm{mg} / \mathrm{kg}$ once per week in cycle $1-2$, every second week in cycle 3-6 and once every 4 weeks thereafter) plus Rd (R: 25 mg on days 1-21; d: 40 mg on days 1, 8, 15 and 22$)(n=368)$ or $R d$ alone ( $n=369)$ until disease progression. ${ }^{4}$ The additional follow-up continued to demonstrate deeper responses with daratumumab plus Rd versus Rd alone at a median follow-up of 56.2 months. Similar to previously published data, the overall response rate (ORR) was $93 \%$ in patients receiving daratumumab plus $\mathrm{Rd}$ and $82 \%$ in patients receiving $\mathrm{Rd}$ alone, with a stringent complete response (SCR) rate of 35\% and 15\%, respectively. In this updated analysis, the significant PFS benefit of daratumumab plus Rd versus Rd alone was maintained. After a median follow-up of 56.2 months, the median PFS for patients receiving daratumumab plus $\mathrm{Rd}$ was not reached, while it was 34.4 months for those receiving Rd alone (HR: 0.53 [95\% Cl:

0.43-0.66]; $p<0.0001)$. The 5-year PFS rate with daratumumabbased regimen was $52.5 \%$ versus $28.7 \%$ with $\mathrm{Rd}$ alone. Furthermore, the median OS was not reached in both treatment arms, with a 5-year OS rate of $66.3 \%$ with daratumumab plus $\mathrm{Rd}$ and 53.1\% with Rd alone (HR: 0.68 [95\% Cl: 0.53-0.86]; $\mathrm{p}=0.0013)$. This benefit is consistent across patient subgroups except for patients with impaired baseline hepatic function. Remarkably, the PFS and OS data have been achieved in a population with $44 \%$ of patients aged between $75-90$ years. Taken together, the updated results strongly support the use of daratumumab plus lenalidomide and dexamethasone as a standard of care for patients with transplant-ineligible NDMM.

\section{Improving clinical outcomes with triplet therapy in RRMM}

At EHA2021, Dr Aurore Perrot reported the updated interim analysis of the efficacy and safety data of the phase III ICARIAMM trial, which assessed isatuximab with pomalidomide and dexamethasone for the treatment of adult patients with relapsed and refractory multiple myeloma (RRMM). The multicenter, open-label, randomized trial included a total of 307 relapsed/ refractory RRMM patients previously treated with $\geq 2$ prior lines of therapies, including lenalidomide and a proteasome inhibitor (PI). Patients underwent 1:1 randomization to receive either isatuximab $(10 \mathrm{mg} / \mathrm{kg}$ once weekly in cycle 1 , then every other week) plus pomalidomide (4 mg on days 1-21) and dexamethasone (20-40 mg once weekly) (Isa-Pd) ( $=154)$ or Pd alone ( $n=153)$, until disease progression or unacceptable toxicity. ${ }^{5}$ The primary endpoint was progression-free survival (PFS), determined by an independent response committee (IRC). This preplanned second interim analysis assessed longer-term outcomes, including time to next treatment (TTNT), overall survival (OS), time from randomization to disease progression on first subsequent therapy or death (PFS2) and safety.

At the time of primary analysis, the trial met its primary endpoint. ${ }^{5}$ At a median follow-up of 11.6 months, Isa-Pd versus Pd was associated with significant improvement in PFS (11.5 months vs 6.5 months; HR: 0.596 [95\% Cl: 0.436-0.814]; $p=0.001$ ). 
At a median follow-up of 35.3 months, Isa-Pd versus Pd alone was associated with significantly prolonged median PFS (11.1 months vs 5.8 months; HR: 0.599; $p<0.0001$ ) and median TTNT (15.5 months vs 8.9 months; HR: 0.555 [95\% Cl: 0.418-0.737]; $\mathrm{p}<0.0001)$. The results further showed that Isa-Pd significantly improved PFS2 (17.51 months vs 12.88 months; HR: 0.759 [95\% Cl: 0.582-0.989]; log-rank $p=0.0202$ ), while there was a strong trend in OS benefit with a 7-month improvement in the median OS (24.57 months vs 17.71 months; HR: 0.760 [95\% $\mathrm{Cl}$ : 0.574-1.008]; log-rank $\mathrm{p}=0.0280) .{ }^{6}$ After additional 2 years of therapy, the responses continued to deepen, with an ORR of $63 \%$ with Isa-Pd versus $33.3 \%$ with Pd alone. Interestingly, this study showed that daratumumab monotherapy following Isa-Pd appears to be less effective than after Pd. However, no difference in response was observed when daratumumab was given as a combination therapy. In conclusion, the longer follow-up confirmed Isa-Pd as a standard of care for patients with RRMM.

\section{Expanding the treatment armamentarium of RRMM}

With the current standard of care therapies in $\mathrm{MM}$, patients who are refractory to the cereblon-targeting drugs lenalidomide and pomalidomide, have only limited options. A novel drug targeting cereblon, iberdomide, has recently proven activity in pomalidomide and lenalidomide refractory myeloma cell lines. ${ }^{7}$ It's efficacy and safety is being assessed in the CC-220-MM-001 study, including a phase I dose-escalation study and a phase II dose expansion study. At the EHA2021, results from cohorts E, F, and G from the phase I, CC-220-MM-001study were presented by Dr Sagar Lonial. Patients included in these cohorts had RRMM with $\geq 2$ prior lines of therapy, including lenalidomide and pomalidomide as well as PIs and whose disease progressed on or within 60 days of last anti-myeloma therapy. Patients were treated with iberdomide (Iber) plus daratumumab (D) plus dexamethasone (d) $(n=43)$ (cohort E), Iber plus bortezomib (V) plus $d(n=25)$ (cohort F) or Iber plus carfilzomib (K) plus d ( $n=9$ ) (cohort G). All patients were heavily pretreated with a median time since diagnosis of $>6.5$ years and $30 \%-40 \%$ of the patients were triple-class refractory. At a median follow-up of 3.9 months for patients treated with IberDd, 5.5 months for those treated with IberVd and 5.1 months for those treated with IberKd, the ORR was 45.9\%, 56.0\%, and 50.0\%, respectively. Of note, the occurrence of non-hematological treatment-emergent adverse events (TEAEs) was low, with fatigue, rash, and gastrointestinal disorders being the most common grade 3-4
TEAEs. The recommended phase II dose (RP2D) is $1.6 \mathrm{mg}$ for patients receiving IberDd, while it was not yet determined in the other cohorts. Overall, the results support further investigation of iberdomide-based combination therapies in phase III studies.

Finally, to further expand the possibilities in MM treatment, novel agents with different mechanisms of action are of great interest. Currently, the bispecific B-cell maturation antigen (BCMA) - and CD3-directed off-the-shelf antibody, teclistamab is evaluated in the first-in-human, multicenter, phase I study MajesTEC-1. ${ }^{8}$ In the open-label, single-arm trial, eligible patients ( $n=157$ ) had measurable MM, were relapsed or refractory or intolerant to established therapies, had not received any prior BCMA-targeted therapy, and fulfilled the following criteria: hemoglobin $\geq 8 \mathrm{~g} / \mathrm{dL}$, platelets $\geq 75 \times 10^{9} / \mathrm{L}$, absolute neutrophil count $\geq 1.0 \times 10^{9} / \mathrm{L}$. Teclistamab was administered intravenously (range $0.3-19.2 \mu \mathrm{g} / \mathrm{kg}$ [once every 2 weeks] or 19.2-720 $\mu \mathrm{g}$ / $\mathrm{kg}$ [once per week]) or subcutaneously (range 80-3000 $\mathrm{kg} /$ $\mathrm{kg}$ [once per week]) in different cohorts, with step-up dosing for $38.4 \mu \mathrm{g} / \mathrm{kg}$ or higher doses. In fact, $1,500 \mathrm{~g} / \mathrm{kg}$ was the determined subcutaneous RP2D. The median number of prior therapies was 5.0 in both the total SC cohort $(n=73)$ as well as the RP2D SC cohort $(n=40)$. Of those patient groups, nearly $80 \%$ were triple-class refractory, $38 \%$ were penta-drugrefractory, and approximately $80 \%$ were refractory to the last line of therapy. Regarding TEAEs, $60 \%$ of patients in the SC total and $70 \%$ of patients in the RP2D cohort experienced cytokine release syndrome (CRS), however, all CRS events were of grade 1 or 2 and therefore manageable. There was only 1 case of neurotoxicity and grade 3-4 cytopenia was generally confined to step-up dosing and in cycles 1 and 2. Infections were reported in 51\% of SC-treated patients, including $21 \%$ of patients experiencing grade 3-4 infections. Keeping in mind that the patient population was heavily pretreated, a promising ORR of $65 \%$ in the RP2D SC cohort was achieved, including $40 \%$ of patients showing $\geq C R$. In patients, who received other SC doses $(n=32)$, an ORR of $59.4 \%$ was achieved. After a median followup of 7.1 months 22/26 (85\%) responders in the RP2D SC group, were still alive and continuing treatment. In conclusion, teclistamab is a well-tolerated agent and no new safety signals were detected. The observed responses were durable and deepened over time, representing a promising starting point for future studies to evaluate teclistamab in earlier-line MM as well as in combination therapies.
1. Leypoldt LB, Besemer B, Asemissen AM et al. Updated Interim Analysis of the GMMG CONCEPT Trial Investigating Isatuximab, Carfilzomib, Lenalidomide, and Dexamethasone (Isa-KRd) in Front-Line Treatment of High-Risk Multiple Myeloma. Presented at the: EHA2021 Virtual Congress; 9-17 June 2021. Oral presentation S183.

2. Garcés J, Cedena MT, Puig N, et al. Circulating Tumor Cells Are The Most Relevant Diagnostic Biomarker in Transplant-eligible Multiple Myeloma. Biomarker in Presented at the: EHA2021 Virtual Congress; 9-17 June 2021. Oral presentation S185.

3. Puig N, Contreras T, Paiva B, et al. Serum Protein Electrophoreisis and Immunofixation vs Mas Spectrometry for Response Assessment in Newly Diagnosed Multiple Myeloma Patients Enrolled in the GEM2021MENOS65 Clinical Trial. Presented at the: EHA2021 Virtual Congress; 9-17 June 2021. Poster presentation EP1012.

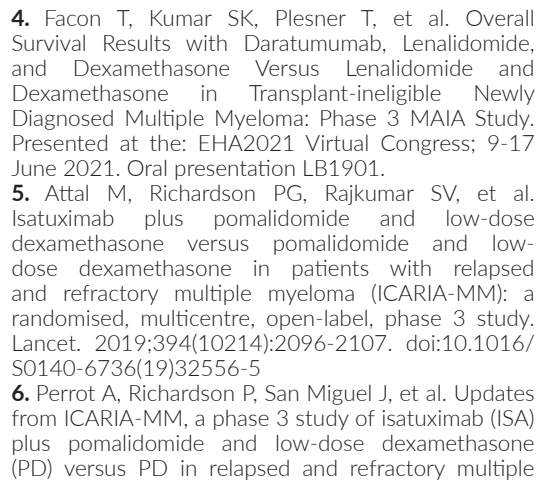

4. Facon T, Kumar SK, Plesner T, et al. Overal Survival Results with Daratumumab, Lenalidomide and Dexamethasone Versus Lenalidomide and Dexamethasone in Transplant-ineligible Newly Diagnosed Multiple Myeloma: Phase 3 MAIA Study. Presented at the: EHA2021 Virtual Congress; 9-17 June 2021. Oral presentation LB1901.

5. Attal M, Richardson PG, Rajkumar SV, et al. Isatuximab plus pomalidomide and low-dose satuximab plus pomalidomididomide and dexamethasone versus pomalidomide and low dose dexamethasone in patients with relapsed and refractory multiple myeloma (ICARIA-MM): a randomised, multicentre, open-label, phase 3 study. Lancet. 2019;394(10214):2096-2107. doi:10.1016/ S0140-6736(19)32556-5

6. Perrot A, Richardson P, San Miguel J, et al. Updates from ICARIA-MM, a phase 3 study of isatuximab (ISA) plus pomalidomide and low-dose dexamethasone (PD) versus PD in relapsed and refractory multiple

myeloma (RRMM). Presented at the: EHA2021 Virtual Congress; 9-17 June 2021. Oral presentation S186. 7. Lonial S, Richardson PG, Popat R, et al. Iberdomide in Combination with Dexamethasone and Daratumumab, Bortezomib, or Carfilzomib in Patients with Relapsed/ Refractory Multiple Myeloma. Presented at the: EHA2021 Virtual Congress; 9-17 June 2021. Oralpresentation S187.

8. van de Donk NWCJ, Garfall AL, Mateos MV et al. Teclistamab a B-cell Maturation Antigen (BCMA) CD3 Bispecific Antibody, in Relapsed/Ref $x$ CD3 Bispecific Antibody, in Relapsed/Refractory Multiple Myeloma: Updated Results of a Phase I, Firstin-human Study. Presented at the: EHA2O21 Virtua Congress; 9-17 June 2021. Oral presentation S193. 